\title{
Avaliação emancipatória de um programa educativo do serviço de controle de infecção hospitalar
}

\author{
Critical evaluation of an infection control educational program \\ Evaluación emancipadora de un programa educativo del servicio de control de infección hospitalaria
}

\section{Danielle Fabiana Cucolo', Josimerci Ittavo Lamana Faria ${ }^{2}$, Claudia Bernardi Cesarino ${ }^{3}$}

\begin{abstract}
RESUMO
Objetivo: Aplicar o modelo da avaliação emancipatória para subsidiar a transformação no Programa Educativo do Serviço de Controle de Infecção Hospitalar de um Hospital de Ensino. Métodos: Trata-se de uma pesquisa-ação, realizada com seis enfermeiros da Unidade de Terapia Intensiva Coronariana de um Hospital de Ensino do interior do Estado de São Paulo. Realizaram-se entrevistas coletivas e nove círculos de discussão fundamentados no método de Paulo Freire. Resultados: Foram identificados 7 temas geradores: programa anual, domínio do conteúdo pelo mediador, horário e duração das aulas, desenvolvimento das aulas, repercussão na prática, instrumentos de avaliação e organização do Serviço de Controle de Infecção Hospitalar. Esta pesquisa possibilitou: maior autonomia dos enfermeiros, integração da equipe de enfermagem nas visitas de vigilância nos diferentes turnos de trabalho e suporte para enfermeiros multiplicadores. Conclusões: $\mathrm{O}$ estudo proporcionou envolvimento dos enfermeiros multiplicadores e subsidiou uma reflexão crítica dos profissionais com estratégias para reestruturação do programa.
\end{abstract}

Descritores: Educação em saúde; Educação em enfermagem; Infecção hospitalar/prevenção \& controle

\section{ABSTRACT}

Objective: To conduct a critical evaluation of an infection control educational program in a Brazilian teaching hospital in order to suggest changes as needed. Methods: This study used action research approach to collect data among 6 nurses working in an Intensive Cardiac Care Unit in a Brazilian teaching hospital in the Sao Paulo State. Paulo Freire's methodology was adopted to conduct interviews and nine discussion sections. Results: Seven themes emerged: establish an annual program; ensure the mediator knows well the content of the program; have a schedule and duration of classes; facilitate class dynamic; focus on evidence-base practice; and, develop an instrument to evaluate the structure and organization of the Infection Control Service. Conclusion: This study involved staff nurses and developed their critical thinking regarding strategies that might be used to re-structure the infection control educational program. In addition, this study also promoted greater nursing autonomy and nursing team cohesion in the control of infection.

Keywords: Health education, Education, nursing; Cross infection/prevention \& control

\section{RESUMEN}

Objetivo: Aplicar el modelo de la evaluación emancipadora para subsidiar la transformación en el Programa Educativo del Servicio de Control de Infección Hospitalaria de un Hospital de Enseñanza. Métodos: Se trata de una investigación-acción, realizada con seis enfermeros de la Unidad de Cuidados Intensivos Coronarios de un Hospital de Enseñanza del interior del Estado de Sao Paulo. Se realizaron entrevistas colectivas y nueve círculos de discusión fundamentados en el método de Paulo Freire. Resultados: Se identificaron 7 temas generadores: programa anual, dominio del contenido por el mediador, horario, duración y desarrollo de las clases, repercusión en la práctica, instrumentos de evaluación y organización del Servicio de Control de Infección Hospitalaria. Esta investigación posibilitó: mayor autonomía de los enfermeros, integración del equipo de enfermería en las visitas de vigilancia en los diferentes turnos de trabajo y soporte para enfermeros multiplicadores. Conclusiones: el estudio favoreció el involucramiento de los enfermeros multiplicadores y subsidió una reflexión crítica de los profesionales con estrategias para la reestructuración del programa.

Descriptores: Educación en salud, Educación en enfermería, Infección hospitalaria/ prevención \& control

${ }^{1}$ Especialista em Enfermagem do Trabalho, responsável pelo Centro de Educação Permanente da Santa Casa de Misericórdia de São José do Rio Preto, São José do Rio Preto (SP), Brasil.

${ }^{2}$ Professora do Departamento de Enfermagem Especializada. da Faculdade de Medicina de São José do Rio Preto. São José do Rio Preto (SP), Brasil.

${ }^{3}$ Professora Departamento de Enfermagem Geral da Faculdade de Medicina de São José do Rio Preto. São José do Rio Preto (SP), Brasil. 


\section{INTRODUÇÃO}

A infecção hospitalar $(\mathrm{IH})$ representa um dos principais problemas da qualidade da assistência à saúde, devido a importante incidência, ao aumento da morbi-mortalidade, dos custos diretos e indiretos, assumindo conseqüências de impacto humano, social e econômico ${ }^{(1-2)}$.

Compete à Comissão de Controle de Infecção Hospitalar $(\mathrm{CCIH})$ elaborar um Programa de Controle de Infecções Hospitalares (PCIH), contendo ações sistematizadas que visem a máxima redução da incidência e da gravidade das IH, implementado pelo Serviço de Controle de Infecção Hospitalar $(\mathrm{SCIH})^{(3)}$.

Inserida no PCIH, a intervenção educativa proporciona maior conhecimento, atitudes positivas por parte dos profissionais da saúde e índices melhores no cumprimento das medidas gerais de prevenção de $\mathrm{IH}^{(4-5)}$.

O enfermeiro assume um importante papel diante dessas ações, uma vez que dentre suas competências e habilidades, ele deve ser capaz de: planejar, implementar e participar dos programas de formação, qualificação contínua e promoção da saúde dos trabalhadores ${ }^{(6)}$.

Nestes programas educacionais, a avaliação da aprendizagem é a principal responsável pela retroalimentação e, portanto, pelo aperfeiçoamento constante do sistema de capacitação e desenvolvimento dos profissionais ${ }^{(7)}$. A avaliação emancipatória visa provocar a crítica de uma dada realidade, fazendo com que as pessoas envolvidas em uma ação educacional gerem as suas próprias alternativas de agir e de transformá-la ${ }^{(8)}$.

Frente à falência dos tradicionais e verticais modelos utilizados pelo SCIH para prevenir e controlar infecção, buscam novas formas de abordar o tema propondo o desenvolvimento conjunto entre profissionais do $\mathrm{SCIH}$ e equipe de enfermagem ${ }^{(9)}$.

Uma importante estratégia utilizada pelos SCIH tem sido a capacitação dos profissionais e o envolvimento dos enfermeiros, tornando-os multiplicadores das ações relacionadas à prevenção de $\mathrm{IH}$, devido a melhor vínculo com a equipe e à supervisão contínua. No entanto, ainda são poucos os serviços que desenvolvem estas atividades e poucos enfermeiros têm uma visão compartilhada sobre a importância das $\mathrm{IH}^{(3)}$.

Considerada a importância das ações educativas e a imprescindível participação dos enfermeiros como multiplicadores e co-responsáveis pela prevenção e controle de IH, vislumbra-se um modelo de avaliação que propicie maior envolvimento desses profissionais, a fim de que nos momentos avaliativos possam expressar os seus entendimentos, suas experiências, opiniões, sugestões e críticas sobre o programa implantado, favorecendo um planejamento conjunto das ações educativas futuras.

Assim, este estudo teve como objetivo aplicar o modelo da avaliação emancipatória para subsidiar a transformação no Programa Educativo do Serviço de Controle de Infecção Hospitalar (PESCIH) de um Hospital de Ensino.

\section{MÉTODOS}

Trata-se de um estudo descritivo de abordagem qualitativa, por apresentar objetivos que necessitam de uma aproximação da realidade vivenciada pelos enfermeiros, com vistas a gerar alternativas e propostas para a melhoria da qualidade do ensino em prevenção e controle de IH.

Foi realizado no ano de 2005 na Unidade de Terapia Intensiva Coronariana de um Hospital de Ensino do interior do Estado de São Paulo e os sujeitos foram os seis enfermeiros desta unidade. Esta pesquisa foi aprovada pelo Comitê de Ética em Pesquisa da Faculdade de Medicina de São José do Rio Preto (Protocolo n ${ }^{\circ} 4198 / 2004$ ) e foi preservada a identidade dos participantes usando-se letras na transcrição dos discursos.

Utilizou-se a pesquisa-ação, pesquisa social empiricamente fundamentada para aprimorar a prática, cujos princípios são: caráter participativo, o impulso democrático e a contribuição para a mudança social. foi desenvolvida em três fases: planejamento, implementação e avaliação $^{(10-11)}$.

Este estudo foi norteado pelo paradigma da avaliação emancipatória, descrito por Saul ${ }^{(8)}$, constituído por três momentos: descrição da realidade, crítica da realidade e criação coletiva. As fases da pesquisa-ação foram operacionalizadas utilizando-se intervenções congruentes com a avaliação emancipatória.

No planejamento, primeira etapa da pesquisa-ação, contemplou-se a preocupação com a temática para o primeiro passo da ação ${ }^{(10)}$, utilizou-se entrevistas coletivas norteadas por questões de ordem pedagógica consoantes com o PESCIH, realizada em quatro círculos de discussão.

Este momento propiciou a verbalização e a problematização de uma realidade vivenciada, de acordo com a apreensão do grupo, contemplando o primeiro momento da avaliação emancipatória. O material obtido desta descrição da realidade, gravado e transcrito, foi categorizado segundo os pressupostos da análise temática de Freire ${ }^{(12)}$ compreendendo o universo temático dos participantes.

O universo temático emerge, quando o indivíduo pensa criticamente o seu mundo que se refere à temática do pensamento dos aprendizes em questão. Buscar o "tema gerador" é saber o que os indivíduos estão pensando a respeito da realidade que estão vivendo ${ }^{(12)}$.

Visualizou-se, assim, após detalhada leitura e seleção das frases repetidas com maior freqüência ou emitidas de forma enfática e congruente com o trabalho educativo, sete temas geradores.

O relato discursivo organizado nos sete temas geradores foi devolvido aos participantes, para análise e crítica da realidade experienciada. Nesta etapa do 
trabalho, já de implementação ${ }^{(10)}$, os sujeitos passam a assumir a sua ação num processo de reflexão da própria prática, possibilitando a reapropriação da realidade e a criação coletiva, integralizada em cinco círculos de discussão, para posterior reelaboração do programa educacional apresentado, totalizando, assim, nove círculos de discussão.

A avaliação, terceira etapa da pesquisa-ação, visualizou se houveram mudanças no PESCIH construídas pelos sujeitos do estudo. Constata-se também as possibilidades e os limites da aplicação da avaliação emancipatória no processo de pesquisa-ação.

\section{RESULTADOS}

Para o planejamento de ensino nos círculos de discussão, foram levantados sete temas geradores: programa anual; domínio do conteúdo pelo mediador; horário e duração das aulas; desenvolvimento das aulas; instrumentos de avaliação; repercussão na prática e organização do SCIH. Estes foram desenvolvidos com os seis enfermeiros, utilizando-se o método de Paulo Freire para conhecer o que esses profissionais pensam do PESCIH, de modo que, ao tomarem consciência, sejam criadores de cultura.

\section{Programa Anual}

$\mathrm{Na}$ realidade analisada, a necessidade de repetir as orientações e retomar sempre as mesmas informações relacionadas à prevenção de $\mathrm{IH}$ foi percebida nas falas: " $O$ processo é válido, a orientação é feita, repete-se na beira do leito todos os dias e a equipe não faz, salvo exceçôes"(C), "Repete sempre”(B), "Isso causa exaustão, entristece, você fica sem saber se é a didática ou qual estratégia usar, éfrustrante"(A). O enfermeiro assistencial nota a falta de integração com o enfermeiro do SCIH, como observa-se no relato: "É necessário chamar e orientar, durante as visitas diárias da CCIH, tanto colaborador quanto o enfermeiro do setor, sobre qualquer anormalidade observada"(C).

\section{Domínio do conteúdo pelo mediador}

O conteúdo abordado deve estar fundamentado e o mediador bem preparado para capacitar o grupo de enfermeiros que serão multiplicadores da aprendizagem. Ao contrário, o descontentamento, a falta de colaboração e credibilidade podem ser reflexo evidente, como citado nos depoimentos: "Quem foi dar a aula não sabia nem do conteúdo que estava falando"(A), "Ninguém conseguiu passar com convicção e fazer aspessoas acreditarem que existe a necessidade de se paramentar..."(C).

A presença mediadora do educador nas situações de aprendizagem ou nas vivências do cotidiano, num processo de diálogo reflexivo, revela sua importância também na área da saúde, como nota-se na fala: "O dia em que en fui ao treinamento, a maioria das pessoas que estavam lá eram de UTI, o que acaba tendo um diferencial dos que trabalham na ala, principalmente para expor sobre Ventilação Mecânica e Pneumonia para quem sabe do que você está falando, tem que ter certeza,...”(C).

\section{Horário e duração das aulas}

É importante ressaltar a pontualidade, respeitando o horário planejado, conforme o discurso: "Tdeal é comesar e terminar no horário previsto"(A) e a abordagem integral do programa educativo, nos três turnos de trabalho: manhã, tarde e noturno, para garantir a continuidade da assistência aliada às medidas preventivas, visto as exteriorizações: "Necessário prestar essa assessoria em todos os turnos..." $(\mathrm{C})$, “O pessoal do noturno se acha excluido"(A), "... o pessoal do noturno ou vem de manhã ou à tarde, se tivesse um horário no noturno seria melhor" $(\mathrm{F})$, "Prejudica a atenção na aula, dependendo do dia e do plantão, se foi muito agitado, é cansativo..."(D).

\section{Desenvolvimento das aulas}

Quando falamos de prevenção e controle das IH, uma questão bastante polêmica está relacionada aos aspectos técnicos: normas, rotinas, padronizações preconizadas pelo SCIH, e a prática da assistência de enfermagem, como vemos a seguir: "Fica claro na exposição, mas na prática sempre tem dívida"(C).

O impacto obtido com dinâmicas, trabalho em grupo e participação dos enfermeiros foi tido como motivador, interessante e despertou curiosidade, de acordo com as falas que se seguem: "Gostei dos questionários"(A), "Sanaram as dúvidas"(E), "Foi dinâmico, objetivo, se ficar maçante o pessoal comega dispersar... algo mais participativo, dinâmico é mais interessante"(D), "Achei boa a estratégia da aplicação dos questionários no início da aula, na prática, você pensa que sabe e quando tem que colocar no papel surgem mil dívidas..."(C).

Outro fator importante para o desenvolvimento do trabalho em grupo é a composição de grupos menores possibilitando a exposição do conhecimento e das dúvidas de todos, assim como expõe as diferenças existentes em cada setor, como foi exteriorizado: "Quanto menor o grupo, melhor para discutir...”(C), “Boa, positiva, participativa, todo mundo participou, interessante"(D), "Esta forma de grupos motiva, estimula a discutir, pois cada unidade tem uma realidade" $(\mathrm{F})$.

\section{Instrumentos de avaliação}

Para acompanhar a adesão e a descentralização das informações relacionadas às boas práticas para controlar $\mathrm{e}$ prevenir $\mathrm{IH}$, o SCIH utilizou instrumentos, tipo check-list, para avaliar aspectos importantes nas diferentes síndromes infecciosas e realizar orientações durante as visitas de rotina nas unidades de internação. Um dos pontos relevantes dos discursos está relacionado à divulgação desses resultados obtidos por meio da avaliação: "... mais interessante é traz̧er os resultados pra gente" $(\mathrm{C})$, "Eles ficarão surpresos com o impacto dos resultados" $(\mathrm{F})$, "Era muito bom quando tínhamos os gráficos, eram deixados nas unidades..."(C).

Outro ponto pertinente a ser abordado é o desconhecimento, pela maioria dos enfermeiros dessa 
unidade, em relação à aplicação pelas enfermeiras do SCIH desses instrumentos avaliativos: "Não conhecia" (C, D e F), "Na realidade, quando en falava do check-list me referia às boas práticas para controle de IH,..."(C), "Não conbecia esta proposta"(B e E).

\section{Repercussão na prática}

A função educadora na enfermagem fica evidente nas falas: "Tá faz.parte da profissão... nossa equipe vai depender da nossa orientação..." $(\mathrm{F})$, "Sempre vêm perguntar para a enfermeira, o que precisam fazer se está em precaução.." (F), assim como, a necessidade de supervisionar e rever sempre as mesmas orientações foi, mais uma vez, ressaltada: "Deu suporte para sermos multiplicadores, mas tem sempre que bater em cima disso"(A), "Tudo o que você cobra acontece de forma mais efetiva, se você deixa de cobrar todo trabalho é perdido"(D).

De forma geral, os enfermeiros exteriorizaram uma sensibilização da equipe de enfermagem após orientações, no entanto, ainda falta comprometimento e envolvimento, assim como faltam recursos humanos e materiais, evidenciados como pontos negativos: "Falta de material é um fator que quebra o processo, dificulta, epara o funcionário é mais cômodo não ter material"(A), "As pessoas sabem, falta envolvimento, comprometimento com o paciente e com a unidade"(B).

\section{Organização do Serviço de Controle de Infecção Hospitalar}

O trabalho em equipe e o consenso sobre as orientações a respeito das padronizações foram relevantes nos discursos: "Importante o grupo da CCIH trabalhar em equipe, falar a mesma lingua"(A), "O grupo fica mais confiante”(C), "A CCIH é a cabeça da instituição, tem que ter o responsável da medicina e da enfermagem, $e$ lá não tem"(C).

\section{DISCUSSÃO}

O método utilizado é sócio-cultural, ativo, dialógico e crítico. Assim, realizou-se uma educação emancipatória e transformadora, em que o elemento norteador foi o diálogo do pesquisador com os sujeitos do estudo, numa relação horizontal.

A aproximação do enfermeiro frente suas atribuições educacionais e as inúmeras dificuldades da prática diária evidenciam que a mera transmissão do conhecimento é insuficiente e não atinge o objetivo de educar e ressaltam a necessidade de capacitação desses profissionais para desempenharem o papel de educadores ${ }^{(13)}$.

A enfermagem é exercida, de forma geral, por um grupo heterogêneo, começando pelo próprio nível de formação, e, nesse sentido, torna-se imprescindível que o enfermeiro assuma a responsabilidade pela educação contínua de sua equipe. Assim, contribuirá para a melhoria da qualidade dos cuidados, para a capacitação dos profissionais e para a valorização dos recursos humanos em saúde ${ }^{(14)}$.

No entanto, ainda existe certo distanciamento dos enfermeiros em relação às práticas educativas, como também uma restrita visão no que se refere aos problemas e necessidades educacionais da equipe de enfermagem ${ }^{(14)}$.

Partindo do princípio de que as instituições de ensino superior são parcialmente responsáveis pela formação, de cidadãos e profissionais competentes, as escolhas das práticas pedagógicas refletem-se na atividade do profissional, mostrando que o distanciamento do enfermeiro da função educativa está relacionado a sua formação(15-16).

A prevenção e o controle de infecção também deve fazer parte da filosofia de formação profissional, assim como, deve fazer parte do processo de educação permanente durante o exercício profissional ${ }^{(17)}$. Os conteúdos sobre IH oferecidos nos cursos de graduação e em outros cursos, muitas vezes, são insuficientes para uma atuação efetiva: faltam conhecimento, clareza e uma visão ampliada de prevenção ${ }^{(18)}$.

O envolvimento do enfermeiro do SCIH nos serviços prestados aos pacientes e colaboradores pode minimizar as dificuldades encontradas para a realização de um trabalho conjunto, objetivando os mesmos ideais ${ }^{(19)}$. Além disso, como, a credibilidade neste educador, o agente de mudanças, também influencia na transformação de atitudes de quem deve implementá-las ${ }^{(20)}$.

Dessa forma, o educador deve favorecer a mobilização dos diferentes saberes priorizando a fala do aprendiz antes de qualquer exposição. Ativar a experiência prévia possibilita ao educando falar do que conhece e sente sobre determinado assunto, e quando tem a oportunidade de revelar-se falando do que conhece, sente e faz, torna-se sujeito integrante da situação-foco, e o processo de ensino-aprendizagem se constrói de forma efetiva $^{(21)}$.

A reflexão sobre a prática educativa tem sido constantemente realimentada e, de acordo com a avaliação emancipatória, permite ao homem, por meio da consciência crítica, imprimir uma nova direção para suas ações, de acordo com valores que elege e com os quais se compromete no decorrer de sua historicidade ${ }^{(8)}$.

A flexibilidade nos horários para realização dos treinamentos tem se mostrado importante, pois quando as programações educativas são desenvolvidas fora do horário de trabalho, torna-se mais difícil a participação dos funcionários e, quando ocorre durante o horário de trabalho, existe a possibilidade de não ser dispensado de suas atividades. É importante ressaltar que o nível de satisfação de cada funcionário reflete a ênfase dada ao desenvolvimento integral do homem como força de trabalho, em sua dignidade absolutat(22).

Considerando o avanço técnico-científico acompanhado do aumento das taxas de infecção, o enfermeiro assistencial vê na CCIH um órgão que, frente às mudanças pode organizar atividades educativas, propiciando a atualização de técnicas e esclarecimento de situações conflitantes entre 
teoria e prática ${ }^{(19)}$.

Sendo assim, os desafios para a implementação de medidas preventivas e de controle de $\mathrm{IH}$ envolvem desde políticas institucionais e administrativas, normatização do serviço, relações interpessoais e intersetoriais, até o envolvimento e a capacitação dos profissionais. Ao enfermeiro do SCIH compete persistência, consciência crítica, capacitação constante e uma incessante busca por habilidades que esclareçam sua prática ${ }^{(20)}$.

Educar é capacitar pessoas para situarem-se responsavelmente no mundo, partilhando idéias e metodologias que valorizem a tomada de posição. É possibilitar ao educando emitir suas opiniões, discutir aspectos positivos e negativos, as dimensões sociais, políticas, éticas, culturais, econômicas, entre outras, caminhando para a formação de cidadãos capazes de pensar e planejar um processo para transformar a sociedade $^{(23)}$.

Quanto à avaliação dos resultados, quando os dados obtidos são entregues apenas ao responsável pelo planejamento dos programas educacionais, a avaliação cumpre uma função de apoio que é externa ao processo de ensino, desconsiderando interesses e necessidades informativas dos participantes que seriam relevantes para o entendimento, interpretação e intervenção de modo mais adequado ${ }^{(8)}$.

Assim, "Não basta termos uma boa idéia ou executarmos um bom trabalbo; é preciso também sermos capazes de fazer com que outras pessoas entendam o que estamos fazendo, porque o fazemos e com que resultado"*

A falta de envolvimento profissional tem dificultado as ações de controle e está relacionada à falta de conscientização acerca da importância dessas medidas, a baixa adesão e ao pouco comprometimento com o serviço e com o paciente ${ }^{(20)}$. Para alcançar a emancipação é necessário fazer uso da consciência crítica, um processo permanente de autoavaliação, e estabelecer um envolvimento de forma responsável e compartilhada para a tomada de decisões democráticas $^{(8)}$.

Os enfermeiros atuantes no SCIH são formalmente responsáveis pela $\mathrm{CCIH}$, porém, tantas vezes, não existe apoio necessário da instituição para o desenvolvimento de suas atribuições, faltando-lhes autonomia para a tomada de decisões. Sendo o enfermeiro do SCIH o elo entre pacientes e profissionais da saúde, a prática exige que o mesmo atue a partir da socialização, desenvolvendo outros valores, como a competência e a confiabilidade ${ }^{(19)}$.

Este estudo oferece subsídios para a prática educativa dos enfermeiros e busca de melhor compreensão, levandoos a uma intervenção transformadora nas medidas de

\footnotetext{
* Barrass R. Os cientistas precisam escrever: guia de redação para cientistas, engenheiros e estudantes. 2a ed. São Paulo: T.A.Queiroz; 1986 apud Silva MJP, Pereira LL, Benko MA. Educaşão continuada: estratégia para o desenvolvimento do pessoal de enfermagem. Rio de Janeiro: Marques Saraiva; São Paulo: Editora da Universidade de São Paulo; 1989.
}

prevenção e controle das IH.

\section{CONSIDERAÇÕES FINAIS}

A aplicação do modelo da avaliação emancipatória com os enfermeiros da Unidade de Terapia Intensiva Coronariana de um Hospital de Ensino do interior do Estado de São Paulo proporcionou a reflexão, a compreensão e a transformação das atividades educativas desenvolvidas pelo $\mathrm{SCIH}$, de forma libertadora.

O método de investigação, a pesquisa-ação, mostrou-se adequado, pois subsidiou a transformação do PESCIH a partir de compromissos sociais e políticos assumidos pelos participantes, numa decisão gerada de forma coletiva e democrática.

Conseguiu-se estabelecer um envolvimento gradativo, já que no desenrolar dos círculos de discussão as exteriorizações foram se ampliando e abrangendo aspectos relacionados ao ambiente institucional, refletindo sobre o SCIH e as relações de poder.

O estudo evidencia a importância da utilização do modelo da avaliação emancipatória proposto por Saul ${ }^{(8)}$, que propiciou a participação dos enfermeiros como seres humanos, ativos, produtivos, tornando-se mais conscientes da importância de seu compromisso na tomada de decisões, transformando, assim, um contexto de trabalho.

As melhorias e recomendações para a prática profissional foram verificadas por meio de maior autonomia dos enfermeiros membros do SCIH, das propostas de aproximação da equipe de enfermagem priorizando a prática educativa durante as visitas de vigilância, presentes nos diferentes turnos de trabalho e dando suporte para os enfermeiros multiplicadores nas ações de prevenção e controle de $\mathrm{IH}$, que se tornaram mais descentralizadas, participativas e criativas. Contudo, o tempo investido na aprendizagem possibilitou, também, muitas mudanças qualitativas no modo de ser do grupo.

Assumindo o papel de avaliador e orientador do trabalho, o pesquisador não encontrou problemas de relacionamento e desenvolveu a experiência em pesquisa e avaliação, visto que a pesquisa-ação permitiu uma integração entre pesquisador e pesquisados, percebida e ampliada ao longo do estudo, estabelecendo um vínculo de responsabilidade e cumplicidade.

Avaliando as limitações do estudo, constatou-se:

- a necessidade de investir maior tempo no planejamento das atividades, para oferecer condições favoráveis ao diálogo, sem haver prejuízo das atividades a serem desenvolvidas na Unidade de Terapia Intensiva;

- o compromisso de não dispersar os círculos de discussão quando havia alguma intercorrência na unidade;

- a necessidade de limitar o tempo de permanência do enfermeiro fora da unidade de internação em trinta a quarenta minutos; 
Não se pretende com este estudo esgotar o assunto e nem buscar "soluções prontas" para a prevenção e controle da IH e, sim, contribuir para o desenvolvimento da enfermagem e oferecer subsídios para o ensino, a prática e pesquisas nesta área, assim como, propiciar a emancipação dos enfermeiros.

\section{REFERÊNCIAS}

1. Sgarbi LPS, Contemo LO. Estrutura e dinâmica das comissões de controle de infecção hospitalar. In: Rodrigues EAC, Mendonça JS, Amarante JMB, Alves Filho MB, Grinbaum RS, Richtman R. Infecções hospitalares: prevenção e controle. São Paulo: Sarvier; 1997. p.37-41.

2. Rodríguez Pérez AU, Sánchez Santos L. Infección nosocomial: impacto y perspectivas. Rev Cuba Hig Epidemiol [periódico na Internet]. 2004 [citado 2005 Dez 11]; 42(2) [ cerca de 5 p.]. Disponível em: http://bvs.sld.cu/revistas.

3. Turrini RNT. Programa de Controle de Infecção Hospitalar: problemas na implementação em hospitais do Município de São Paulo. Acta Paul Enfermagem. 2004; 17(3): 316-24.

4. Arévalo RH, Cruz MR, Palomino VF, Fernández VF, Guzmán RE, Melgar AR. Aplicación de un programa de control de infecciones intrahospitalarias en estabelecimientos de salud de la Región San Martín, Perú. Rev Peru Med Exp Salud Publica. 2003;20(2):84-91.

5. Cuéllar Ponce de León L, Rosales CR, Aquiño RF. Eficacia de un programa educativo para la prevención y el control de infecciones intrahospitalarias en el Instituto Especializado de Enfermedades Neoplásicas, Lima, Perú. Rev Peru Med Exp Salud Publica. 2004; 21(1): 37-43.

6. Fernandes CNS. Refletindo sobre o aprendizado do papel de educador no processo de formação do enfermeiro. Rev Latinoam Enfermagem. 2004; 12(4): 691-3.

7. Borges-Andrade JE. Desenvolvimento de medidas em avaliação de treinamento. Estud Pscicol (Natal). 2002; 7(Esp): 31-43.

8. Saul AM. Avaliação emancipatória: desafio à teoria e à prática de avaliação e reformulação de currículo. 6a ed. São Paulo: Cortez; 2001.

9. Cruz EDA. A formação de um elo: uma proposta de enfermagem para a prevenção das infecções hospitalares. Cogitare
Enferm. 2001; 6(1): 14-24.

10. Tripp D. Pesquisa-ação: uma introdução metodológica. Educ Pesqui. 2005; 31(3):443-66.

11. Santos JBG. Avaliação emancipatória: uma alternativa para a facilitação da aprendizagem na disciplina enfermagem em centro cirúrgico [tese]. Ribeirão Preto: Escola de Enfermagem de Ribeirão Preto da Universidade de São Paulo; 1996.

12. Freire P. Pedagogia do oprimido. 22a ed. Rio de Janeiro: Paz e Terra; 1993.

13. Cadete MMM. A concretude da atividade educativa do enfermeiro. Mundo Saúde (1995). 2000; 24(5): 380-6.

14. Darvim RMB, Torres GV, Santos SR. Educação continuada em enfermagem: conhecimentos, atividades e barreiras encontradas em uma maternidade escola. Rev Latinoam Enfermagem. 1999; 7(5): 43-9.

15. Faria JIL, Casagrande LDR. A educação para o século XXI e a formação do professor reflexivo na enfermagem. Rev Latinoam Enfermagem. 2004; 12(5): 821-7.

16. Villa EA, Cadete MMM. Capacitação pedagógica: uma construção significativa para o aluno de graduação. Rev Latinoam Enfermagem. 2001; 9(1): 53-8.

17. Tipple AFV, Pereira MS, Hayashida M, Moriya TM, Souza ACS. O ensino do controle de infecção: um ensaio teóricoprático. Rev Latinoam Enfermagem. 2003; 11(2): 245-50.

18. Santos IF. Enfermeiro e a infecção hospitalar: um estudo sobre sua formação e atuação [tese]. Ribeirão Preto: Escola de Enfermagem de Ribeirão Preto da Universidade de São Paulo; 1997.

19. Alves DCI, Évora YDM. Questões éticas envolvidas na prática profissional de enfermeiros da comissão de controle de infecção hospitalar. Rev Latinoam Enfermagem. 2002; 10(3): 265-75.

20. Souza ACS, Tipple AFV, Pereira MS, Prado MA. Desafios para o controle de infecção nas instituições de saúde: percepção dos enfermeiros. Cienc Enferm. 2002; 8(1):19-30.

21. Freire P. Pedagogia da autonomia: saberes necessários à prática educativa. 16ª ed. São Paulo: Paz e Terra; 1996.

22. Silva MJP, Pereira LL, Benko MA. Educação continuada: estratégia para o desenvolvimento do pessoal de enfermagem. Rio de Janeiro: Marques Saraiva; São Paulo: Editora da Universidade de São Paulo, 1989.

23. Gomes JB, Casagrande LDR. A educação reflexiva na pósmodernidade: uma revisão bibliográfica. Rev Latinoam Enfermagem. 2002; 10(5): 696-703. 